Acta bot. bras. 1(2):257-262 (1988) supl.

\title{
FITOSSOCIOLOGIA DA MATA DO MORRO DA QUIXABA NO TERRITÓRIO DE FERNANDO DE NORONHA.
}

\author{
Manoel Cláudio da Silva Júnior (1) \\ Jeanine Maria Felfili (1) \\ Carolyn Elinore Barnes Proença (2) \\ Ana Cristina Miranda Brasileiro (3) \\ Benício de Melo Filho \\ Paulo Ernane Nogueira da Silva \\ Kátia Lemos Costa
}

\begin{abstract}
RESUMO - O território de Fernando de Noronha localiza-se a $32^{\circ} 25^{\prime} 30^{\prime \prime}$ W de longitude e 3 $50^{\prime} 10^{\prime \prime} \mathrm{S}$ de latitude e é composto por 21 ilhas. Este trabalho foi realizado na ilha principal na região do Morro da Quixaba até a Ponta da Sapata, onde a vegetação foi menos perturbada nas últimas décadas. Utilizou-se o método de quadrantes para a amostragem de indivíduos com diâmetro mínimo de $5 \mathrm{~cm}$ a $30 \mathrm{~cm}$ do solo. A distância entre os pontos foi estabelecida $\mathrm{em} 10 \mathrm{~m}$. No total foram aplicados 100 pontos de amostragem, em diversas picadas alocadas na área de estudo. Foram encontradas 13 famílias sendo dominantes, Nyctaginaceae, Bignoniaceae, Anacardiaceae, Rubiaceae e Euphorbiaceae. Dentre as 16 espécies encontradas, Guapira sp. ocupou a primeira posição em importância com IVI de 100,67\%, seguida de Tabebuia roseoalba $(55,14 \%)$, Spondias mombin $(52,17 \%)$, Guettarda sp. $(21,72 \%)$ e Allophylus sp. $(17,27 \%)$.
\end{abstract}

\begin{abstract}
Fernando de Noronha territory is located at $32^{\prime \prime} 25^{\prime} 30^{\prime \prime} \mathrm{W}$ and $3^{\prime \prime} 50^{\prime} 10^{\prime \prime} \mathrm{S}$ and it is composed of 2.1 islands. We studied the Quixaba Hill wihch extends until to Sapata Point, where the vegetation has been relatively undisturbed on the last decades. The method used the point-centered-quarter. Only individuals with, at least, $5 \mathrm{~cm}$ diameter above the ground level was sampled. The distance between points was $10 \mathrm{~m}$ with points on peackies in the studied area. We obtained 16 species distributed in 13 families with the dominance of Nyctaginaceae, standing out Bignoniaceae, Anacardiaceae, Rubiaceae and Euphorbiaceae. Guapira sp. reached the best IVI (100,67), followed by Tabebuia roseo-alba $(55,14)$, Spondias mombin $(52,17)$, Guettarda sp. $(21,72)$ e Allophylus sp. $(17,27)$.
\end{abstract}

Key-words: Phytosociology, Floristics, Fernando de Noronha.

\section{Introdução}

No Brasil há uma carência de estudos fitossociológicos em todas as suas formações. Estes são fundamentais para o reconhecimento da composição florística atual e da importância das diferentes espécies na vegetação. Os resultados destes estudos são subsídios importantes para o estabelecimento da área de distribuição das espécies, bem como para o estudo do relacionamento da vegetação com as características ambiente. Além disso, a longo prazo, pode-se avaliar a sucessão vegetal, pela realização contínua de levantamentos na mesma área, considerando-se o aparecimento de novas espécies e também a variação do posicionamento destas espécies em função do índice do valor de importância (IVI).

(1) Departamento de Engenharia Florestal - Universidade de Brasília-DF. C.P. 152807. CEP 70919.

(2) Jardim Botânico de Brasília - CNPq.

(3) Embrapa - Cenargem. 
Os ecossistemas insulares, por serem geograficamente isolados, são de grande valor para a realização destes estudos, visando o esclarecimento dos processos de colonização e extinção das espécies vegetais (Macarthus \& Wilson 1967).

O Arquipélago de Fernando de Noronha apresenta um grande potencial para aqueles estudos, entretanto poucos foram os trabalhos realizados nesse local. Apenas Ridley (1891) apresentou uma lista detalhada das espécies da vegetação do arquipélago, não havendo, porém, quantificação e nenhum acompanhamento das mudanças na vegetação.

O objetivo básico deste trabalho foi estudar a fitossociologia da formação florestal do Morro da Quixaba; efetuar uma comparação qualitativa com os resultados de Ridley (1891) e obter subsídios para futuras propostas de zoneamento ambiental.

\section{Material e Métodos}

O Arquipélago de Fernando de Noronha localiza-se a $32^{\circ} 25^{\prime} 30^{\prime \prime}$ W e $3^{\circ} 50^{\prime} 10^{\prime \prime} \mathrm{S}$ e dista cerca de $540 \mathrm{~km}$ de Recife, sendo composto por 21 ilhas.

Este trabalho foi realizado na ilha Principal, no Morro da Quixaba até a Ponta da Sapata, onde a vegetação foi menos alterada nas últimas décadas.

Para a amostragem dos indivíduos com diâmetro mínimo de tronco de $5 \mathrm{~cm}$ a $30 \mathrm{~cm}$ do solo, aplicou-se o método de quadrantes (Cottam \& Curtis 1956) com 100 pontos de amostragem, distantes entre si em $10 \mathrm{~m}$ e dispostos em várias picadas alocadas aleatoriamente na área de estudo.

Para os cálculos dos parâmetros fitossociológicos ioram utilizadas as seguintes fórmulas, conforme Mueller-Dombois \& Ellemberg (1974) e Mártins (1979):

Densidade Relativa (DR) $\ldots \ldots \ldots$ DR $(\%)=\frac{n i}{N} \times 100$

Área Basal Relativa $(A B R) \ldots \ldots A B R(\%)=\frac{A B i}{A B} \times 100$

Freqüência Absoluta $(F A) \ldots \ldots \ldots \overline{F A}=\frac{P i}{P} \times 100$

Freqüência Relativa (FR) $\ldots \ldots \ldots \ldots F R=\frac{F A i}{F A} \times 100$

İndice do Valor de Importância (IVI) .. IVI = DR+ABR+FR

İndice de Diversidade de Shannon \& Weaver $\left(\mathrm{H}^{\prime}\right) \ldots \ldots$. .

$$
H^{\prime}=-\sum_{i=1}^{S} p i \text { In pi }
$$

onde:

ni = número de indivíduos da espécie $\mathrm{i} ; \mathrm{N}=$ número total de indivíduos amostrados; $\mathrm{ABi}=$ área basal da espécie $\mathrm{i} ; \mathrm{AB}=$ área basal de todas as espécies; $\mathrm{Pi}=$ número de pontos em que a espécie $\mathrm{i}$ ocorreu; $\mathrm{P}=$ número total de pontos aplicados; $\mathrm{FA}=$ freqüência absoluta da espécie i; FA = freqüência absoluta de todas as espécies. 
Conforme sugestão de Gibbs et al. (1980), após o término da amostragem foi feita uma vistoria geral na área para a observação das espécies raras.

Para todas as espécies encontradas, foi coletado material botânico para identificação, que foi feita por especialistas. Este material encontra-se depositado no Herbário da Universidade de Brasilia (UB).

\section{Resultados e Discussão}

Segundo Martins (1979) o predomínio numérico dos individuos de uma familia, expressa a dominância de familias. Entre os 400 individuos amostrados destacaram-se na vegetação estudada as cinco familias representadas na Figura 1, somando 245,16\% de IVI o que representa $81,72 \%$ do total.

Para as familias Nyctaginaceae, Bignoniaceae, Anacardiaceae e Rubiaceae foi amostrada apenas uma espécie para cada. A familia Euphorbiaceae contou com 3 espécies.

Entre as outras familias encontradas apenas Leguminosae tinha 2 espécies (1 Caesalpinoideae e 1 Mimosoideae) as demais, Sapindaceae, Capparidaceae, Theophrastaceae, Sapotaceae, Annonaceae, Celastraceae e Myrtaceae contaram com apenas 1 espécie.

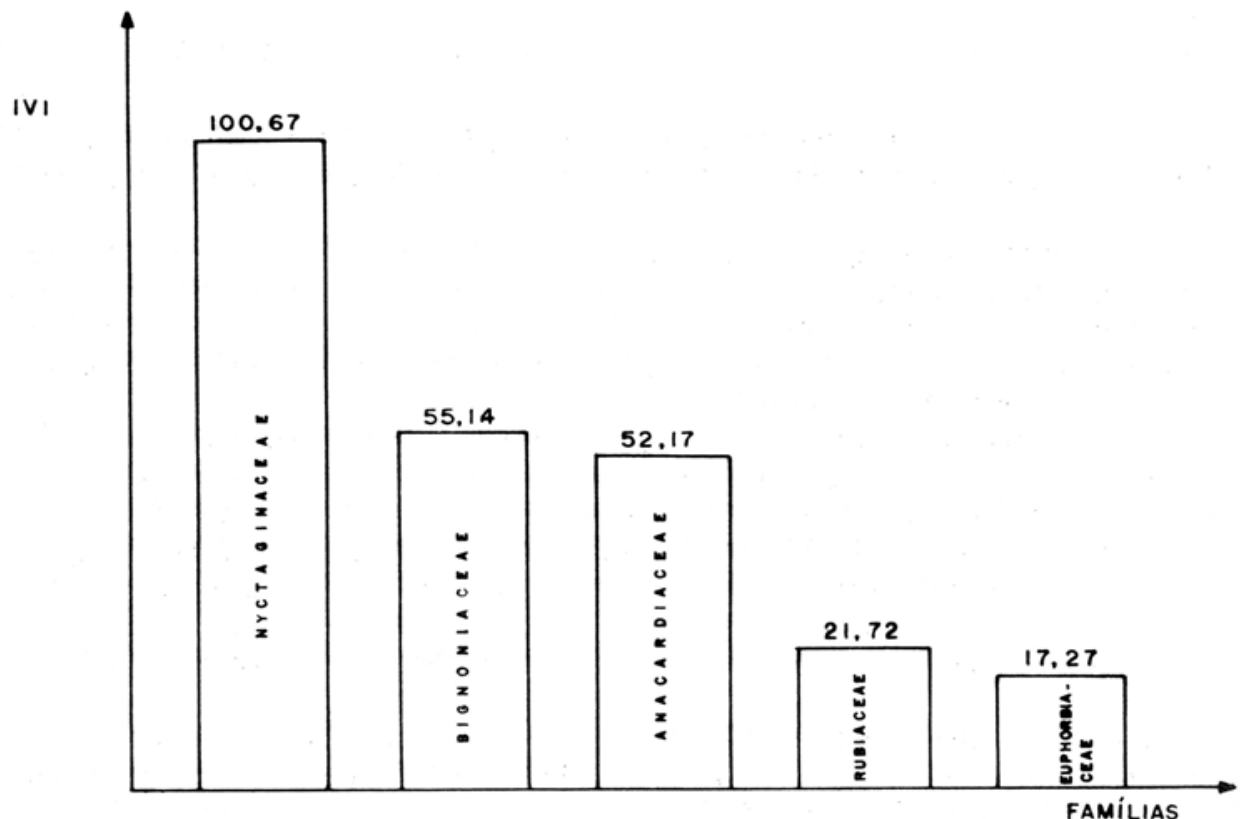

Figura 1 - Indice do valor de Importância (IVI) das familias. 
A análise do número de indivíduos por espécie, a frequência relativa, a densidade relativa e a área basal relativa é representada na Tabela 1.

Guapira sp. ocupou a primeira posição com 33,55\% do IVI total. Segundo Ridley (1891) esta espécie apresentava-se como arbustos e árvores muito comuns na ilha Principal.

TABELA 1: Espécies amostradas na região do Morro da Quixaba, em r'c mando de Noronha, apresentadas em ordem decrescente do índice do valor de Importância (IVI). Onde: $\mathrm{N}=$ número de indivíduos, $\mathrm{FR}=$ freqüência relativa, $\mathrm{DR}=$ densidade relativa, $\mathrm{ABR}=$ área basal relativa.

\begin{tabular}{llrrrrr}
\hline Espécie & Familia & N & FR & DR & ABR & IVI \\
\hline Guapira sp. & Nyctaginaceae & 145 & 26,14 & 36,25 & 38,28 & 100,67 \\
Tabebuia roseo-alba (Ridley) Sawd & Bignoniaceae & 69 & 18,56 & 17,25 & 19,33 & 55,14 \\
Spondias mombin L. & Anacardiaceae & 76 & 17,80 & 19,00 & 15,37 & 52,17 \\
Guettarda sp. & Rubiaceae & 25 & 8,33 & 6,25 & 7,13 & 21,72 \\
Allophylus sp. & Sapindaceae & 17 & 6,44 & 4,25 & 4,77 & 15,46 \\
Sapium sceleratum Ridley & Euphorbiaceae & 18 & 6,44 & 4,50 & 2,89 & 13,83 \\
Capparis flexuosa Blume ex K. Hassk. & Capparidaceae & 12 & 4,55 & 3,00 & 3,04 & 10,58 \\
Erythrina velutina Willd. var. aurantiaca & Leguminosae-Pap. & 09 & 2,65 & 2,25 & 1,58 & 6,48 \\
Jacquinia armillaris Jacq. & Theophrastaceae & 06 & 1,89 & 1,50 & 2,21 & 5,61 \\
Bumelia fragrans Ridley & Sapotaceae & 06 & 1,89 & 1,50 & 1,50 & 4,89 \\
Annona squamosa L. & Annonaceae & 05 & 1,14 & 1,25 & 1,22 & 3,61 \\
Maytenus cf. nitida Mart. & Celastraceae & 04 & 1,14 & 1,00 & 1,04 & 3,18 \\
Jatropha pohliana Muell. Arg. & Euphorbiaceae & 03 & 1,14 & 0,75 & 0,71 & 2,59 \\
Eugenia ilhensis Berg. & Myrtaceae & 03 & 1,14 & 0,75 & 0,68 & 2,57 \\
Savia sp. & Euphorbiaceae & 01 & 0,38 & 0,25 & 0,22 & 0,85 \\
Acacia farnesiana Willd. & Leguminosae-Mim. & 01 & 0,38 & 0,25 & 0,01 & 0,64 \\
& & & & & &
\end{tabular}

Guapira sp. ocupou a primeira posição com $33,55 \%$ do IVI total. Segundo Ridley (1891) esta espécie apresentava-se como arbustos e árvores muito comuns na ilha Principal.

Em observação da regeneração natural, constatou-se muitos indivíduos de Guapira sp., provenientes de brotação de raízes. Neste sentido, estudos mais detalhados forneceriam informações quanto ao sucesso de estabelecimento desta espécie via sementes, o que poderia indicar se a reprodução vegetativa é uma alternativa de colonização face à problemas de polinização, germinação, etc.

Para Tabebuia roseo-alba, que ocupou a segunda posição em importância, o valor de IVI obtido foi um pouco maior do que a metade do valor da primeira colocada. Os comentários de Ridley (1891) indicam-na como uma espécie arbórea muito abundante na Ponta da Sapata, que é a parte terminal da região da Quixaba onde a presente amostragem foi realizada. Comparativamente com as demais espécies encontradas, Tabebuia roseo-alba, é a única que apresenta sementes com adaptaçōes para a dispersão por vento. Parece pouco provável que suas sementes tenham chegado a itha sem a intervenção hu. mana.

Spondias mombin, embora apresente um número de indivíduos maior que o da segunda colocada, obteve a $3^{\text {a }}$ posição em importância devido aos valores menores dos diâmetros dos seus indivíduos, comparativamente àqueles dos indivíduos de Tabebuia roseo-alba. Para Ridley (1891) esta espécie ocorre em grande número na Ponta da Sapata, sugerindo que esta é uma espécie selvagem e que taivez tenha sido trazida por pássaros.

A mesma interpretação anterior pode ser dada em relação ao posicionamento das espécies Allophylus $\mathrm{sp}$. e Sapium sceleratum que respectivamente ocuparam a $5^{\mathrm{a}}$ e $6^{\mathrm{a}}$ posições em importância. O parâmetro que determinou a $5^{a}$ posição de Allophylus sp. fol a 
área basal relativa (ABR). Esta espécie citada por Ridley (1891) como Schimidelia insulana foi tida como muito abundante na ponta da Sapata, principalmente nas áreas abertas.

Para Capparis flexuosa, que obteve a $7^{\mathrm{a}}$ posição em IVI e apresentou uma área basal relativa maior que a de Sapium sceleratum (6ª posição em IVI), o parâmetro da densidade relativa (DR) definiu a posição obtida.

Quanto a importância de cada parâmetro na determinação do posicionamento das espécies, Martins (1979) salienta que a densidade relativa (número de individuos) ou a área basal relativa (porte dos individuos) têm preponderância e que os valores da freqüência relativa (distribuição dos individuos na área) seguem de perto os valores da densidade relativa (DR).

A "burra leiteira", como é chamada a espécie Sapium sceleratum, é uma das grandes árvores da ilha, podendo atingir até $20 \mathrm{~m}$ de altura, seu exsudado leitoso é tido como cáustico, provocando ferimentos mesmo no couro de animais. Segundo Ridley (1891) seus frutos servem de alimento para pequenas aves, que dispersam suas sementes por toda a ilha. Para os ilhéus, seu número aumentou bastante quando as cabras, que constituem a principal criação do local, permaneciam soltas, uma vez que aquelas não se alimentavam das mudas desta espécie devida ao seu exsudado.

Entre as demais espécies é interessante ressaltar Erythrina velutina var. aurantiaca. endêmica em Fernando de Noronha que segundo Ridley (1891), aparentemente estaria em processo de extinção, porém, nesta amostragem, embora em pequeno número os indivi . duos apresentavam grande porte, além disso em observaçōes da regeneração foram encontradas muitas mudas estabelecidas.

Além das espécies apresentadas na Tabela 1, foram encontradas algumas outras, de porte arbóreos, possivelmente nativas, que não foram incluidos na amostragem.

No Morro da Quixaba, Capparis cynophallophora foi encontrada em grande número como arbusto, apenas 1 individuo apresentava diâmetro superior a $5 \mathrm{~cm}$. Também foram encontradas com apenas um individuo, Bauhinia forficata, e uma Rubiaceae desconhecida.

No Morro do Francês, 1 individuo de Talisia sp. foi encontrado, e nas encostas pedregosas alguns exemplares de Ficus noronhac de qrandes dimensōes.

As outras espécies arbóreas encontradas na ilha foram, pela sua localização, consideradas introduzidas pelo homem, como por exemplo Sterculia sp., Artocarpus sp. Cassia grandis, Moquilea tome'ntosa, Ficus lambertiana. Mangifera indica etc.

Apesar de muitos relatórios citarem Fernando de Noronha como um paraíso de flora riquíssima, a vegetação arbórea estudada apreşentou-se com baixa diversidade, como pode ser comprovado pelo índice de Shannon \& W'Eaver, calculado em 'H' = 1,95. Esta é uma característica esperada para os ecossistemas insulares, onde a colonização natural depende da forma de dispersão das sem€ntes, dos agentes de dispersão, da viabilidade das sementes, da distância co centro de dispersão, além de outros fatores.

Este pequeno número de espécies arbóreas nativas indica um ecossistema frágil, principalmente quando se considera o grande número de espécies invasoras lá existentes. Esta situação exige que qualquer intervenção seja baseada em $\epsilon$ studos detalhados, para que não se coloque $€ \mathrm{~m}$ risco a regeneração das matas.

Atualmente já se constata nas bordaduras e nas trithas no interior da mata um grande número de invasoras, onde grupos de árvores apresentem-se totalmente cobertos por Ipomcea spp.

\section{Conclusões}

O estudo fitossociológico da Mata de Mcrro da Quixaba, demonstrou ser a diversidade floristica pequena, uma vez que, apenas 16 espécies arbóreas foram amostradas. Somente umá espécie obteve máis de um terço do IVI total e as quatro espécies má.is im- 
portantes somaram $78,75 \%$ do número total de indivíduos amostrados.

Estes parâmetros sugerem um ecossistema frágil e que devido às dificuldades para a sua colonização natural, pode ser facilmente alterado, através de intervençōes sem a realização de estudos mais detalhados como a avaliação do potencial de regeneração natural, dos problemas de biologia reprodutiva, da capacidade de reprodução vegetativa, da dinâmica de populações e do estado nutricional das espécies. Estas informaçōes seriam subsídios importantes para a elaboração de um plano de manejo para a área estudada.

A região do Morro da Quixaba até Ponta da Sapata embora apresente áreas com ruínas de construçōes antigas é a região menos perturbada nas últimas décadas, devendo ser considerada prioritária na seleção de áreas de preservação. Cabe ainda a definição do tipo de unidade de preservação que deve ser implementada, uma vez que os ilhéus se utilizam de algumas espécies para a obtenção de produtos de sua necessidade.

\section{Referências Bibliográficas}

COTTAN, G. \& CURTIS, J.T. 1956. The use of distance measurements in phytosociological sampling. Ecology 37: 451-460.

GIBBS, P.E.; LEITĀO FILHO, H.F. \& ABBOT, R.J. 1980. Application of the point-centered quarter method in a floristic survey of an área of gallery forest near Mogi-Guaçu. State of São Paulo, SE. BRAZIL. Revta bras. Bot. 3: 17-22.

MAcARTHUR, R.H. \& WILSON, E.O. 1967. The theory of island biogeography, Princeton, Princeton University press. 203p.

MARTINS, F.R., 1979. O método de quadrantes e a fitossociologia de uma floresta residual no interior do Estado de São Paulo: Parque Estadual de Vassununga. São Paulo, Universidade de São Paulo, 239 p. (Tese D.S.)

MUELLER-DOMBOIS, D. \& ELLEMBERG, M. 1974. Aims and methods in vegetation ecology. New York, Willey and Sons, $547 \mathrm{p}$.

RIDLEY, H.N. 1981. Notes of the botany of Fernando de Noronha. Jour. Linn. Soc. (Botany) 27: 1-95. 